\title{
Manual zu Sportverletzungen
}

Das Buch ist das offizielle Manual der Gesellschaft für Orthopädisch-Traumatologische Sportmedizin. Die GOTS ist der weltweit zweitgrösste Zusammenschluss von Sportorthopäden und Sporttraumatologen, die Athleten zum einen wissenschaftlich begleiten, zum anderen medizinisch betreuen. Der Gesellschaft, die Sportmediziner aus der Schweiz, Österreich und Deutschland vereinigt, gehören fast 1000 Mitglieder an.

Das Buch gibt keine praxisnahen, direkten Hinweise zur Behandlung von Sportverletzungen. Es bietet weit mehr. Das Thema wird umfangreich und mit grossem Hintergrundwissen aufgearbeitet. Selbst auf Begleitmassnahmen wie Ernährung, Kleidung und orthopädische Hilfsmittel wird eingegangen.

Nach der Vorstellung allgemeiner Prinzipien der Sportmedizin und diagnostischer Verfahren werden im dritten Kapitel die Sportverletzungen nach Körperregionen gegliedert besprochen. Der Schwerpunkt des Buches liegt jedoch auf den sportartspezifischen Verletzungen. In diesem Abschnitt werden die einzelnen Sportarten mit ihren Besonderheiten, geschichtlichen Hintergründen, Ausrüstungsmaterial und Techniken vorgestellt, sowie auf die sportartspezifischen Anforderungen, Unfallmechanismen, Verletzungen und Überlastungsschäden eingegangen. Eswerden Behandlungs-und Präventionsmassnahmen erörtert. Weil es in keinem Sportmedizinbuch fehlen sollte, wird dem Thema erlaubte und verbotene Wirkstoffe und Medikamente das Kapitel «Medikamente und Sport» gewidmet. Es enthält auch Hinweise auf die Dopingliste.

Es ist den Autoren gut gelungen, Informationen hinsichtlich der gegebenen Relevanz auszuwählen und sie dann logisch und übersichtlich aufgearbeitet zu präsentieren. Das Werk ist vor allem Ärzten und Trainern, aber auch allen anderen, die sich für fundiertes sportmedizinisches Hintergrundwissen interessieren, zu empfehlen. Die unzähligen Literaturangaben zeugen vom wissenschaftlichen Anspruch des Buches. Bei gezielten Fragestellungen lassen sich mit Hilfe des 28-seitigen Registers und des gut gegliederten Inhaltsverzeichnisses alle Themenbereiche leicht finden. Sie sind gut gegliedert und der Leser muss bestimmte Themen nicht lange suchen. Es fallen der sehr übersichtliche Aufbau und die anschaulichen Fotos auf. Die meisten Abbildungen sind schwarz-weiss, aber wo es sinnvoll und notwendig ist, finden sich Farbfotografien, die in der Mitte des Buches auf 16 Seiten zusammengefasst sind.

Dr. med. Sandra Krüger, Berlin

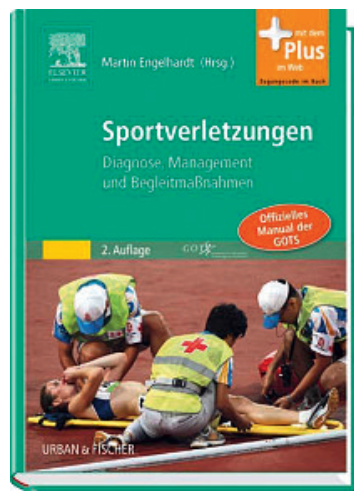

Martin Engelhardt (Hrsg.) Sportverletzungen

Offizielles Manual der GOTS

München:

Urban \& Fischer

bei Elsevier; 2009.

2. Aufl., 864 Seiten.

$160 \mathrm{CHF}$.

ISBN 978-3-437-24091-1 